\title{
System with Distributed Lag: Adaptive Identification and Prediction
}

\author{
Nikolay Karabutov \\ Moscow state university of information technology, radio engineering, electronics/Department of \\ Problems Control; Moscow, Russia \\ E-mail:kn22@yandex.ru, nik.karabutov@gmail.com
}

\begin{abstract}
Adaptive algorithms of parametric identification of discrete systems with lag variables are proposed. Adaptive algorithms (AA) in the presence of lag input variables are developed. The convergence of the AA and the boundedness of the trajectories the adaptive system is proved. Convergence domain AA depends on operating disturbance. Models with multiplicative parameters (MPM) for the decrease of a number estimated parameters are offered. The process for selection of the vector of base parameters MPM was developed. The performance of adaptive system identification for this case is proved. It is shown that parameters of system estimation at the application of multiplicative identification must be chosen from a condition of minimization of the criterion of the prediction error. Transformation of interdependence between the lagged variables is offered, allowing eliminating their effect on system work. In the second part of work, the method of synthesis AA identification of the systems containing lagged output variables is offered. We consider a case of linear correlation between an output of the system and operating disturbance. For a solution of a problem, we suggest fulfilling an estimation of operating disturbance. Corresponding procedures are described and proved their efficiency. Simulation results are presented that confirm the efficiency of the adaptive methods.
\end{abstract}

Index Terms-Adaptive algorithm, identification, distributed lag, convergence, model, decision making.

\section{INTRODUCTION}

Lag models are used to a description of work engineering [1-3] and economic systems [4-7], and also in medicine [8-10]. Accounting lag variables leads to several problems that the solution of the problem parametric identification complicated. The main of these problems is the relationship (correlation, multicollinearity) between variables [4-6]. In economic systems, this interaction arises between the dependent and independent variables and the operating disturbances. Various models of approximation of parameters at distributed lags (parametric schemes) are applied to attenuation of such interferences on the identification process. This reduces the number of estimated parameters of the object.

In identification systems widely apply the parametric the Коуск scheme $[5,11]$. It is based on a change of coefficients of a model on a decreasing geometric progression.
Fisher model $[4,12]$ is based on the change of model coefficients for a given decreasing arithmetic progression. Arithmetical and geometrical models are applied, when plant parameters decrease from the first members of a progression [13]. Considering it, S. Almon [14] modified Fisher model. He has applied the polynomial law of a change of factors. In [12] parametric scheme based on the use of logarithmic normal distribution. This same concept based on the application of exponential distribution law, is considered in [15]. In [16] applied the Pascal distribution, which leads to a simple form for the coefficients. A priori define of relationship in the parametric scheme in the form of a rational polynomial is considered in [17]. Other approaches to define of factors at distributed lags are considered in [7]

Application of parametric schemes in systems with a distributed lag is a dominating direction in an econometrics. Usually, various modifications specified above schemes for the purpose of decrease of a number of estimated parameters [18] are applied. The main purpose of such schemes is the identification of parameters with the help a method of least squares or a maximum likelihood [4, 18]. In conditions of a priori uncertainty, this approach to identification is not always effective and requires additional time-consuming research. For a solution of the problems inherent parametric schemes, models of adaptive or rational expectations [19] apply. Parametric schemes the problem of consistent estimation on finite samples generates. Considering these difficulties, application of adaptive methods is the perspective approach to an estimation of parameters.

The adaptive approach is applied for the synthesis of models describing the change of economic indicators. So in [20] the expanded Kalman filter is applied. The adaptive algorithm of tuning parameters of the filter is recursive procedure of least squares. Note that the authors of the concept of "adaptive" can attach different meanings. Adaptation [21] can be a choice of structure model on the basis of the application search of the set candidates. In [22] proposed a heuristic procedure, which is interpreted as adaptive. In [23] apply the least squares method for tuning parameters of a linear adaptive model with a distributed lag on the input. The parametric scheme of a change of factors of the model is set A priori. It allows to minimize the number of adjusted parameters. The structure of model by results of simulation is chosen. In [24] the problem of an estimation parameters system with a 
linear distributed lag on an independent variable is considered. It is assumed that the model coefficients decrease with increasing lag. The solution of a problem an estimation parameters on the basis of quadratic functional minimization is searched. Two-step procedure of identification is offered. On the first step, Fletcher-Reeves version of the iterative gradient procedure is applied. On the second step the obtained estimations of parameters are substituted new on the basis of an application of piecewise monotonic approximation. All works on models with distributed lag statistical interpretation of input and output variables used. In the majority of works objects with a scalar input are considered.

So we see that identification of the parameters of systems with distributed lag in most cases on the use of various parametric schemes is based. The use of such schemes estimates the number of unknown parameters reduces. Such approach is not effective at minimization of a parametric error (PE) between model and system parameters. For minimization PE the various approaches based on a transformation of lag variables, apply. In the conditions of a priori uncertainty this approach preliminary simulation for formation of the initial scheme of a change of parameters demands. The formed scheme further correction and improvement of initial assumptions demands. Therefore, the problem of minimization PE within this paradigm is not always correct.

We propose an adaptive approach to the identification of parameters system with a vector input and not make assumptions about the law of variation of model coefficients. The obtained result on the application of a functional-multiple approach to the problem of identification is based. We do not make assumptions concerning statistical properties of input and lag variables, and also disturbances. We suppose that the restricted disturbance acts on a system and vectors of an input and an output system are piecewise-restricted functions. First, an autoregressive model is considered and given the conditions of convergence of the adaptive algorithms. Further, the case of the presence of lag variables on an input variable is examined. At this stage, we solve a problem decrease of the influence of correlations between lag variables on properties of adaptive algorithm. We for a problem solution offer the special transformation of lag variables. The adaptive model with the multiplicative parameters belonging to the restricted area is considered. We show how to choose the set of base parameters of the model, and give conditions for the convergence of the algorithms for this case. In the final part of the paper, we consider the case when the relationship between the disturbance and the output variable of the system exists. Conditions detection of this relationship is adduced. The adaptive observer of disturbance for ensure of convergence of adaptive algorithms is introduced into identification system. The problem of structural identification of system with lag variables inprocess is not considered. The results obtained in [25, 26], are applied for its solution. We give development of the results obtained in [27].

\section{PROBLEM STATEMENT}

Consider the system described by the equation

$$
y_{n}=A^{T} U_{n}+B^{T} X_{n}+\xi_{n},
$$

where $y_{n} \in R$ is an output; $U_{n} \in R^{k}$ is the input vector which elements are piecewise-restricted of functions a time; $n \in J_{N}=[0, N]$ is a discrete time, $N<\infty$; $X_{n}=X\left(u_{i, n}, y_{n}\right) \in R^{m}$ is a vector of distributed lags on and $y_{n}, X_{n}=\left[X_{u_{i, n}}^{T}, X_{y_{n}}^{T}\right]^{T} ; A \in R^{k}, B \in R^{m}$ are vectors of fixed parameters; $\xi_{n} \in R$ is external disturbance, $\left|\xi_{n}\right|<\infty$ for all $n \in J_{N}$.

For (1) we have set of the measured values

$$
\mathrm{I}_{o}=\left\{y_{n}, U_{n} \forall n \in J_{N}\right\}
$$

and the map $\Gamma_{o}:\left\{U_{n}\right\} \times\left\{y_{n}\right\} \quad \forall n \in J_{N}$ describing an observable informational portrait [7, 19].

Problem: estimate set of parameters system (1) on the basis of the analysis $\mathrm{I}_{o}, \Gamma_{o}$

Consider at first a case, when a vector

$$
X_{i, n}=X_{u_{i, n}}=\left[u_{i, n-1}, u_{i, n-2}, \ldots, u_{i, n-m}\right]^{T} \in R^{m},
$$

where lags are only on an input variable $u_{i, n} \in U_{n}$.

We suppose that the lag structure on $u_{i, n}$ is defined by means of the approach offered in [25].

\section{ADAPTIVE IDENTIFICATION OF SYSTEM (1) WITH LAG ON INPUT}

Consider lag presence in an input variable $u_{i, n} \in U_{n}$. We obtain a vector $X_{i, n}=X_{u_{i, n}}, X_{i, n} \in R^{m}$. We will consider various variants of correlation between elements of a vector $X_{i, n} \in R^{m}$ and we will obtain algorithms of identification parameters model.

\section{A. Lack of correlation between lags}

Apply to identification of parameters of system (1) model

$$
\hat{y}_{n}=\hat{A}_{n-1}^{T} U_{n}+\hat{B}_{n-1}^{T} X_{i, n},
$$

where $\hat{y}_{n} \in R$ is a output of model; $\hat{A}_{n-1} \in R^{k}, \hat{B}_{n-1} \in R^{m}$ are vectors of adjusted parameters of model.

Let $e_{n}=y_{n}-\hat{y}_{n}$ is an error of prediction an output of the system (1). It satisfies the equation 


$$
e_{n}=\delta A_{n-1}^{T} U_{n}+\delta \hat{B}_{n-1}^{T} X_{i, n}+\xi_{n}
$$

where $\delta A_{n}=A_{n}-\hat{A}_{n}, \delta B_{n}=B_{n}-\hat{B}_{n}$ are parametric errors.

Problem: estimate parameters of the system (1) by means of the model (3) on the basis of analysis the set $\mathrm{I}_{o}$, minimizing criterion $V_{e, n}=e_{n}^{2}$.

Consider Lyapunov function (LF) $V_{e, n}=e_{n}^{2}$. Define adaptive algorithm from stability conditions of system (1), (4)

$$
\Delta V_{e, n}=V_{e, n}-V_{e, n-1} \leq 0
$$

Differentiate $\Delta V_{n}$ on $\delta A_{n}$ and $\delta B_{n}$. Then

$$
\begin{gathered}
\delta A_{n}=\delta A_{n-1}-\Gamma_{A} e_{n} U_{n}, \\
\delta B_{n}=\delta B_{n-1}-\Gamma_{B} e_{n} X_{i, n},
\end{gathered}
$$

where $\Gamma_{A} \in R^{k \times k}, \Gamma_{B} \in R^{m \times m}$ are diagonal matrixes with $\gamma_{i i, A}>0, \gamma_{j j, B}>0$. Matrixes $\Gamma_{A}, \Gamma_{B}$ convergence of algorithms (5), (6) in the presence of disturbances ensure.

From (5), (6) we adaptive algorithms of adjustment parameters of model (3) we obtain

$$
\begin{aligned}
& \hat{A}_{n}=\hat{A}_{n-1}+\Gamma_{A} e_{n} U_{n}, \\
& \hat{B}_{n}=\hat{B}_{n-1}+\Gamma_{B} e_{n} X_{i, n} .
\end{aligned}
$$

Let

$\delta H_{n}=\left[\delta A_{n}^{T}, \delta B_{n}^{T}\right]^{T}, K_{n}=\left[U_{n}^{T}, X_{i, n}^{T}\right]^{T}, \Gamma=\Gamma_{A} \dot{+} \Gamma_{B}$.

Then for $\delta H_{n}$ obtain algorithm

$$
\delta H_{n}=\delta H_{n-1}-\Gamma e_{n} K_{n}
$$

where $\dot{+}$ is a sign of the direct sum of matrixes.

\section{Assumptions.}

1. The vector $K_{n} \in R^{k+m}$ is extreme nondegenerate, restricted and

$$
\alpha_{0} I_{(k+m)} \leq K_{n} K_{n}^{T} \leq \alpha_{1} I_{(k+m)},
$$

where $\alpha_{0}, \alpha_{1}$ are some nonnegative numbers, $I_{k+m} \in R^{(k+m) \times(k+m)}$ is unity matrix.

2. $\left\|K_{n}\right\|^{2} \leq \beta^{2}<\infty$ for $\forall n \geq 0$.
The convergence (7), (8) follows from following statement [27].

\section{Theorem 1. Let:}

1) disturbance $\xi_{n}$ in (1) is limited for $\forall n \geq 0$ : $\left|\xi_{n}\right| \leq v$, where $v \geq 0$ is some number;

2) there are functions $V_{e, n}, V_{\delta, n}=\left\|\delta H_{n}\right\|^{2}$, where $\|\cdot\|$ is a vector norm;

3) $\tilde{\gamma} I_{k+m} \leq \Gamma \leq \bar{\gamma} I_{k+m} \quad, \quad$ where $\tilde{\gamma}=\lambda_{\min }(\Gamma), \bar{\gamma}=\lambda_{\text {max }}(\Gamma)$ are minimum and maximum eigenvalues of the matrix $\Gamma$;

4) vectors $U_{n}, X_{i, n}$ are the piecewise continuous functions $n$, satisfy to assumptions 1,2 .

Then all trajectories of system (1), (3), (5), (6) are restricted and true estimations

$$
V_{\delta, n} \leq \frac{\kappa v^{2}}{1-\mu}, V_{e, n} \leq \frac{\kappa_{\xi} v^{2}}{\kappa_{e}}
$$

If

$$
\tilde{\gamma} \alpha_{0} \leq 1+\bar{\gamma}^{2} \beta^{4}, 0<\kappa_{e}<1
$$

where $\mu=1-\tilde{\gamma} \alpha_{0}+\bar{\gamma}^{2} \beta^{4}, \beta^{2}\left(\frac{\pi^{2}}{\rho}+2 \bar{\gamma}^{2}\right)=\kappa,\|\Gamma\| \leq \pi$, $K_{n}^{T} \Gamma K_{n} \leq \vartheta<\infty, \quad\left|K_{n-1}^{T} \Gamma K_{n}\right| \leq \tilde{\vartheta}<\infty \quad, \quad \kappa_{\xi}=\vartheta+\tilde{\vartheta}$, $\kappa_{e}=1+\vartheta-2 \tilde{\vartheta}-\theta,, \theta>0$.

Proof. Consider LF $V_{\delta, n}=\left\|\delta H_{n}\right\|^{2}$. Considering (9), $\Delta V_{\delta, n}$ we write in the form

$$
\Delta V_{\delta, n}=-2 e_{n} \delta H_{n-1}^{T} \Gamma K_{n}+e_{n}^{2} K_{n}^{T} \Gamma^{2} K_{n}
$$

Let assumptions 1, 2 are fulfilled. As $\Gamma \leq \bar{\gamma} I_{k+m}$, for $e_{n}^{2} K_{n}^{T} \Gamma^{2} K_{n}$ we obtain $e_{n}^{2} K_{n}^{T} \Gamma^{2} K_{n} \leq e_{n}^{2} \bar{\gamma}^{2} \beta^{2}$. As $e_{n}$ fulfils (4) then an augend in the right part (11) write as

$$
-2 e_{n} \delta H_{n-1}^{T} \Gamma K_{n}=-2 \delta H_{n-1}^{T} \Gamma K_{n} K_{n}^{T} \delta H_{n-1}-2 \xi_{n} \delta H_{n-1}^{T} \Gamma K_{n} .
$$

Then

$$
-2 e_{n} \delta H_{n-1}^{T} \Gamma K_{n} \leq-2 \tilde{\gamma} \alpha_{0} V_{\delta, n-1}+2 \beta v\|\Gamma\|\left\|\delta H_{n-1}\right\| .
$$

Let $\|\Gamma\| \leq \pi, \pi>0$ is some number. Then, after simple transformations we obtain for $\Delta V_{\delta, n}$

$$
\begin{aligned}
\Delta V_{\delta, n} \leq & -2 \tilde{\gamma} \alpha_{0} V_{\delta, n-1}+2 \beta v\|\Gamma\|\left\|\delta H_{n-1}\right\|+e_{n}^{2} \bar{\gamma}^{2}\left\|K_{n}\right\|^{2} \\
& \leq-2 \tilde{\gamma} \alpha_{0} V_{\delta, n-1}+2 \beta v \pi \sqrt{V_{\delta, n-1}}+2 \bar{\gamma}^{2} \beta^{2}\left(V_{\delta, n-1} \beta^{2}+v^{2}\right) .
\end{aligned}
$$


So,

$\Delta V_{\delta, n} \leq-2\left(\tilde{\gamma} \alpha_{0}-\bar{\gamma}^{2} \beta^{4}\right) V_{\delta, n-1}+2 \beta v \pi \sqrt{V_{\delta, n-1}}+2 \bar{\gamma}^{2} \beta^{2} v^{2}$.

We apply the inequality [29]

$$
-a z^{2}+b z \leq-\frac{-a z^{2}}{2}+\frac{b^{2}}{2 a}, \quad a>0, \quad b \geq 0, \quad z \geq 0 .
$$

Then

$\Delta V_{\delta, n} \leq-\left(\tilde{\gamma} \alpha_{0}-\bar{\gamma}^{2} \beta^{4}\right) V_{\delta, n-1}+\frac{\beta^{2} v^{2} \pi^{2}}{\tilde{\gamma} \alpha_{0}-\bar{\gamma}^{2} \beta^{4}}+2 \bar{\gamma}^{2} \beta^{2} v^{2}$.

Let $\tilde{\gamma} \alpha_{0}-\bar{\gamma}^{2} \beta^{4}=\rho$. Then

$$
\Delta V_{\delta, n} \leq-\rho V_{\delta, n-1}+\beta^{2} v^{2}\left(\frac{\pi^{2}}{\rho}+2 \bar{\gamma}^{2}\right)
$$

We obtain the condition of convergence algorithms (7), (8) from $\Delta V_{\delta, n} \leq 0$

$$
\tilde{\gamma} \alpha_{0} \leq 1+\bar{\gamma}^{2} \beta^{4}
$$

Introduce designations

$$
1-\rho=\mu, \beta^{2}\left(\frac{\pi^{2}}{\rho}+2 \bar{\gamma}^{2}\right)=\kappa .
$$

Then for $V_{\delta, n}$ we have inequalities

$$
V_{\delta, n} \leq \mu V_{\delta, n-1}+v^{2} \kappa
$$

Iterating in (13) $n$ from $n-1$ to 0 , for sufficiently large $n$ for $V_{\delta, n}$ we obtain an estimation

$$
V_{\delta, n} \leq \mu^{n} V_{\delta, 0}+v^{2} \kappa \sum_{i=0}^{n} \mu^{i} \leq \frac{v^{2} \kappa}{1-\mu}=\frac{v^{2} \kappa}{\rho} .
$$

Find an estimation for $e_{n}$. Write down expression for $\Delta V_{e, n}=e_{n}^{2}-e_{n-1}^{2}$

$$
\Delta V_{e, n-1}=\left(\delta H_{n-1}^{T} K_{n}\right)^{2}+2 \xi_{n} \delta H_{n-1}^{T} K_{n}+\xi_{n}^{2}-e_{n-1}^{2} .
$$

Considering (9), transform an augend in (14) to a form

$$
\left(\delta H_{n-1}^{T} K_{n}\right)^{2}=\left(\delta H_{n-2}^{T} K_{n}\right)^{2}-2 e_{n-1} \delta H_{n-2}^{T} K_{n-1} z^{2}+e_{n-1}^{2} \tilde{z},
$$

where $z_{n}^{2}=K_{n}^{T} \Gamma K_{n}, \tilde{z}_{n}=K_{n-1}^{T} \Gamma K_{n}$.
As $\delta H_{n-2}^{T} K_{n}=e_{n-1}-\xi_{n-1}$, then

$$
\begin{aligned}
\left(\delta H_{n-1}^{T} K_{n}\right)^{2}= & \left(\delta H_{n-2}^{T} K_{n}\right)^{2}-2 e_{n-1}^{2} z_{n}^{2} \\
& +2 e_{n-1} \xi_{n-1} z_{n}^{2}+e_{n-1}^{2} \tilde{z}_{n}
\end{aligned}
$$

Substitute (15) in (14). Then we have

$$
\begin{aligned}
\Delta V_{e, n-1}= & \left(\delta H_{n-2}^{T} K_{n}\right)^{2}-2 e_{n-1}^{2} z_{n}^{2} \\
& +2 e_{n-1} \xi_{n-1} z_{n}^{2}+e_{n-1}^{2} \tilde{z}_{n} \\
& +2 \xi_{n} \delta H_{n-1}^{T} K_{n}+\xi_{n}^{2}-e_{n-1}^{2} .
\end{aligned}
$$

Apply conditions of the theorem 1. We have $z_{n}^{2} \leq \vartheta,\left|\tilde{z}_{n}\right| \leq \tilde{\vartheta}$ for $\forall n>0$, where $\vartheta>0, \tilde{\vartheta}>0$. Then we will transform (16) in a form

$$
\begin{aligned}
\Delta V_{e, n-1}= & -e_{n-1}^{2}(1+2 \vartheta-\tilde{\vartheta})+\left(\delta H_{n-2}^{T} K_{n}\right)^{2} \\
& +2 e_{n-1} \xi_{n-1} z_{n}^{2}+2 \xi_{n} \delta H_{n-1}^{T} K_{n}+\xi_{n}^{2} .
\end{aligned}
$$

Use the equation (9) and write $\xi_{n} \delta H_{n-1}^{T} K_{n}$ in a form

$$
\xi_{n} \delta H_{n-1}^{T} K_{n}=\xi_{n}\left(\delta H_{n-2}^{T} K_{n}-e_{n-1} \tilde{z}_{n}\right) .
$$

Substitute (18) in (17). Let exists such $\theta>0$ that

$$
\left(\delta H_{n-2}^{T} K_{n}\right)^{2}+2 \xi_{n} \delta H_{n-2}^{T} K_{n}+\xi_{n}^{2} \leq \theta e_{n-1}^{2} .
$$

Then we will obtain

$$
\begin{aligned}
\Delta V_{e, n-1} & =-(1+2 \vartheta-\tilde{\vartheta}-\theta) e_{n-1}^{2}+2 e_{n-1} \xi_{n-1} z_{n}^{2} \\
& -2 \xi_{n} e_{n-1} \tilde{z}_{n} \leq-(1+2 \vartheta-\tilde{\vartheta}-\theta) e_{n-1}^{2} \\
& +\left(e_{n-1}^{2}+v^{2}\right)(\vartheta+\tilde{\vartheta}) .
\end{aligned}
$$

Let $\kappa_{e}=1+\vartheta-2 \tilde{\vartheta}-\theta, \kappa_{\xi}=\vartheta+\tilde{\vartheta}$. We suppose that $0<\kappa_{e}<1$. Then

$$
\Delta V_{e, n-1} \leq-\kappa_{e} V_{e, n-1}+\kappa_{\xi} v^{2}
$$

We from this inequality obtain

$$
V_{e, n} \leq \eta V_{e, n-1}+\kappa_{\xi} v^{2}
$$

where $\eta=1-\kappa_{e}$.

Use the approach stated above. Then

$$
V_{e, n} \leq \frac{\kappa_{\xi} v^{2}}{\kappa_{e}}
$$


So, boundedness of trajectories of system is proved.

The theorem 1 condition convergence of algorithms (7), (8) at execution of the condition (10) gives. Limiting properties of the obtained estimations of parameters and an error $e_{n}$ from a power of operating disturbance $\xi_{n}$ depend. If the condition (10) is not fulfilled, to an assessment of works of adaptive system apply the approach offered in [28]. Properties of algorithms depend on an initial estimate.

Consider an informational power [3] of disturbances $\xi_{n}$

$$
\Xi_{\xi}=\frac{1}{N} \sum_{i=1}^{N} \xi_{i}^{2}
$$

If $\xi_{n}$ is a random noise with a zero average, then $\Xi_{\xi}$ is a variance $\xi_{n}$. In this case the theorem 1 known result about an asymptotic estimation for parameters of the system (1) gives.

Consider a case when the condition (10) is not fulfilled. In this case various a priori premises about the law of a change elements vector $B$ in (1) use. These assumptions do not always correspond to the real system parameters. We in an adaptive system have parametric disturbances. These disturbances must be compensated. Existing approaches we reviewed in the introduction. Next, we present an approach that does not require such assumptions.

\section{B. Multiplicative adaptive algorithm}

We describe an approach that allows the number of adjustable parameters of the model for the system (1) for the case $X_{i, n}=X_{u_{i, n}}$ to be reduced. It uses a posteriori estimates for the vector $H_{B}=H$ introduced in section 3.A. These estimations are the basis for construction of the base model system. Next we for the purpose of obtaining of adequate mathematical model modify a base model.

We determine the number $m$ of lag variables by using the method proposed in $[25,26]$. Apply the following algorithm $A H_{B}$ of formation a vector $H_{B}$.

Algorithm $A H_{B}$.

1. Apply a method of least squares (LSM) and define parameters of secants $y_{n}$ on $x_{j, n} \in X_{i, n}, u_{\mathrm{i}, n} \in U_{n}$

$$
\begin{gathered}
\gamma_{y, x_{j, n}}=a_{y, j, 0}+a_{y, j, 1} x_{j, n}, 1 \leq j \leq m, \\
\gamma_{y, u_{l, n}}=a_{y, l, 0}+a_{y, l, 1} u_{l, n}, 1 \leq l \leq k
\end{gathered}
$$

and coefficients of determination $r_{y, x_{j, n}}^{2}, r_{y, u_{l, n}}^{2}$.

2. Generate the variable $s_{n}=I^{T} U_{n}$, construct model

$$
\hat{y}_{s, n}=\hat{a}_{s, 0}+\hat{a}_{s, 1} s_{n},
$$

and define its parameters by means of LSM.

3. Introduce a variable $\varepsilon_{n}=\hat{y}_{s, n}-y_{n}$ and for it define secants

$$
\gamma_{\varepsilon, x_{j, n}}=a_{\varepsilon, j, 0}+a_{\varepsilon, j, 1} \varepsilon_{n}, 1 \leq j \leq m
$$

and coefficients of determination $r_{y, x_{j, n}}^{2}$.

4. Generate a vector $\tilde{H}_{B}$ on the basis of sets $\left\{a_{\varepsilon, j, 1}\right\}$, $\left\{a_{y, j, 1}\right\},\left\{a_{y, l, 1}\right\}$. Fulfil selection of coefficients at lag variables and $u_{i, n}$, applied the following rule

If $r_{y, x_{j, n}}^{2} \leq r_{\varepsilon, x_{j, n}}^{2}$, then $a_{\varepsilon, j, 1} \in \tilde{H}_{B}$, otherwise $a_{y, j, 1} \in \tilde{H}_{B}$. Apply this rule to choice of the coefficient at $u_{i, n}$.

5. Construct model $y_{B, n}=\tilde{H}_{B}^{T} K_{n}$ and obtain the forecast $y_{n}$.

6. Compute mean values $\bar{y}_{n}, \bar{y}_{B, n}$ for $y_{n}, y_{B, n}$ and informational powers $\Xi_{y}, \Xi_{y_{B}}$ (19).

7. Introduce numbers $\varphi \geq 0, \varphi_{B} \geq 0$. If

$$
\left(\left|\bar{y}-\bar{y}_{B}\right| \leq \varphi\right) \&\left(\left|\Xi_{y}-\Xi_{y_{B}}\right| \leq \varphi_{B}\right)
$$

then suppose $H_{B}=\tilde{H}_{B}$ and consider $H_{B}$ as a base vector of the model. End of algorithm.

8. If (20) it is false, then fulfil correction of elements $\tilde{H}_{B}$.

Consider adaptive multiplicative model

$$
\hat{y}_{B, n}=\hat{\eta}_{n-1} H_{B}^{T} K_{n},
$$

where $\hat{\eta}_{n} \in R$ is the adjusted parameter of model, $\hat{y}_{B, n}$ is a model output. Apply algorithm $A H_{B}$ and define a vector $H_{B} \in R^{k+m}$.

Designate an error of prediction an output system (1) as $e_{B, n}=y_{n}-\hat{y}_{B, n}$. Consider LF $V_{e_{B}, n}=e_{B, n}^{2}$. From stability conditions of system (1), (21) obtain algorithm of adjustment $\hat{\eta}_{n}$

$$
\hat{\eta}_{n}=\hat{\eta}_{n-1}+\gamma_{\eta} e_{B, n} H_{B}^{T} K_{n}=\hat{\eta}_{n-1}+\gamma_{\eta} e_{B, n} \kappa_{n},
$$

where $\gamma_{\eta}>0$ is a parameter ensuring convergence (21), $\kappa_{n}=H_{B}^{T} K_{n}$.

We consider that $\hat{\eta}_{n} \leq 1$. The algorithm (22) ensures criterion minimization

$$
V_{\delta, B, n}=\left\|H-\hat{\eta}_{n} H_{B}\right\|^{2} .
$$


Write down expression for a prediction error $e_{B, n}$

$$
e_{B, n}=\delta H_{B, n-1}^{T} K_{n}+\xi_{n},
$$

where $\delta H_{B, n-1}=H-\hat{\eta}_{n-1} H_{B}$.

Conditions of convergence algorithm (22).

Theorem 2. Let:

i) there is a vector $H_{B}$ obtained by means of algorithm $A H_{B}$;

ii) there are functions $V_{e, n}, V_{\delta, n}=\left\|\delta H_{n}\right\|^{2}$, where $\|\cdot\|$ is a vector norm;

iii) assumptions 1, 2 are fulfilled;

iiii) disturbance $\xi_{n}$ in (1) is limited for $\forall n \geq 0$ : $\left|\xi_{n}\right| \leq v$, where $v \geq 0$ is some number.

Then the algorithm (22) converges at

$$
\gamma_{\eta} \leq \frac{e_{B, n}^{2}-v^{2}}{e_{B, n}^{2} \beta^{2}}
$$

and for $V_{\delta, n}$ the estimation is true

$$
V_{\delta, n} \leq \frac{\kappa_{v} v^{2}}{\gamma_{\eta}\left(\alpha_{0}-\gamma_{\eta} \beta^{4}\right)},
$$

if

$$
\begin{gathered}
\alpha_{0}>\gamma_{\eta} \beta^{4}, \gamma_{\eta}\left(\alpha_{0}-\gamma_{\eta} \beta^{4}\right) \leq 1, \\
\kappa_{v}=\frac{\gamma_{\eta} \beta^{2}\left(1+2 \gamma_{\eta}\left(\alpha_{0}-\gamma_{\eta} \beta^{4}\right)\right)}{\left(\alpha_{0}-\gamma_{\eta} \beta^{4}\right)} .
\end{gathered}
$$

Proof. Consider LF $V_{\delta, n}=\left\|\delta H_{B, n}\right\|^{2}$. As

$$
\delta H_{B, n}=\delta H_{B, n-1}-\gamma_{\eta} e_{\eta, n} K_{n},
$$

then

$$
\begin{aligned}
\Delta V_{\delta, n} & =-2 \gamma_{\eta} e_{B, n} \delta H_{B, n-1}^{T} K_{n}+\gamma_{\eta}^{2} e_{B, n}^{2}\left\|K_{n}\right\|^{2} \\
& =-2 \gamma_{\eta} e_{B, n}^{2}+2 \gamma_{\eta} e_{B, n} \xi_{n}+\gamma_{\eta}^{2} e_{B, n}^{2}\left\|K_{n}\right\|^{2} .
\end{aligned}
$$

As $2 \gamma_{\eta} e_{B, n} \xi_{n} \leq \gamma_{\eta}\left(e_{B, n}^{2}+v^{2}\right), \Delta V_{\delta, n}$ we will transform to a form

$$
\Delta V_{\delta, n} \leq-\gamma_{\eta}\left(e_{B, n}^{2}-v^{2}\right)+\gamma_{\eta}^{2} e_{B, n}^{2} \beta^{2} .
$$

The algorithm (22) will be convergence, if

$$
-\gamma_{\eta}\left(e_{B, n}^{2}-v^{2}\right)+\gamma_{\eta}^{2} e_{B, n}^{2} \beta^{2} \leq 0 .
$$

We obtain a condition of convergence of algorithm from this inequality

$$
\gamma_{\eta} \leq \frac{e_{B, n}^{2}-v^{2}}{e_{B, n}^{2} \beta^{2}} .
$$

The estimation for $\gamma_{\eta}$ is fair, if $e_{B, n}^{2} \geq v^{2}$.

The procedure of obtaining of estimation (23) in the proof of the theorem 1 is stated. We will obtain estimation (23). Substitute in (24) equation for $e_{B, n}$. Then we have

$$
\begin{aligned}
& \Delta V_{\delta, n}=-2 \gamma_{\eta}\left(\delta H_{B, n-1}^{T} K_{n}+\xi_{n}\right) \delta H_{B, n-1}^{T} K_{n} \\
&+ \gamma_{\eta}^{2}\left(\delta H_{B, n-1}^{T} K_{n}+\xi_{n}\right)^{2}\left\|K_{n}\right\|^{2} \\
& \leq-2 \gamma_{\eta} \alpha_{0} V_{\delta, n-1}-2 \gamma_{\eta} \xi_{n} \delta H_{B, n-1}^{T} K_{n} \\
&+ \gamma_{\eta}^{2}\left(\delta H_{B, n-1}^{T} K_{n}+\xi_{n}\right)^{2} \beta^{2} . \\
& \text { As }\left(\delta H_{B, n-1}^{T} K_{n}+\xi_{n}\right)^{2} \leq 2\left(\beta^{2} V_{\delta, n-1}+v^{2}\right), \text { then } \\
& \Delta V_{\delta, n} \leq-2 \gamma_{\eta} \alpha_{0} V_{\delta, n-1}+2 \gamma_{\eta} \nu \beta \sqrt{V_{\delta, n-1}} \\
&+2 \gamma_{\eta}^{2}\left(\beta^{2} V_{\delta, n-1}+v^{2}\right) \beta^{2}= \\
&=-2 \gamma_{\eta}\left(\alpha_{0}-\gamma_{\eta} \beta^{4}\right) V_{\delta, n-1} \\
&+2 \gamma_{\eta} \nu \beta \sqrt{V_{\delta, n-1}}+2 \gamma_{\eta}^{2} \nu^{2} \beta^{2} .
\end{aligned}
$$

We suppose that $\alpha_{0} \geq \gamma_{\eta} \beta^{4}$. Then for $\Delta V_{\delta, n}$ is obtained

$$
\begin{aligned}
\Delta V_{\delta, n} \leq-\gamma_{\eta}\left(\alpha_{0}-\gamma_{\eta} \beta^{4}\right) V_{\delta, n-1} \\
+\frac{\gamma_{\eta} \nu^{2} \beta^{2}\left(1+2 \gamma_{\eta}\left(\alpha_{0}-\gamma_{\eta} \beta^{4}\right)\right)}{\left(\alpha_{0}-\gamma_{\eta} \beta^{4}\right)}
\end{aligned}
$$

From (25) obtain an inequality for $V_{\delta, n}$

$$
V_{\delta, n} \leq(1-\rho) V_{\delta, n-1}+\kappa_{v} v^{2},
$$

when $\rho=\gamma_{\eta}\left(\alpha_{0}-\gamma_{\eta} \beta^{4}\right)$,

$$
\kappa_{\nu}=\frac{\gamma_{\eta} \beta^{2}\left(1+2 \gamma_{\eta}\left(\alpha_{0}-\gamma_{\eta} \beta^{4}\right)\right)}{\left(\alpha_{0}-\gamma_{\eta} \beta^{4}\right)} .
$$

At $n \rightarrow \infty$ we obtain estimation (23).

From theorems 1, 2 follows that the algorithms (7), (8) if the condition is true (10) give a true estimates of the parameters of the system (1) at $\xi_{n}=0$. This conclusion 
follows from this that adaptive procedures are difference equations of the first order of adjustment parameters model (4). This statement is true for the model (21) with the algorithm (22).

The adaptive algorithm (22) at an operation $\xi_{n}$ ensures execution a condition $\left|e_{B, n}\right| \leq \delta_{\eta}\left(\xi_{n}\right)$ where $\delta_{\eta}>0$ is some specified value. If this condition is not fulfilled, transform model (21), for example, to a form

$$
\hat{y}_{B, n}=\left[\hat{\eta}_{1, n-1}, \hat{\eta}_{2, n-1}\right]^{T} \mathcal{H}\left(H_{B}\right) K_{n}
$$

where $\mathcal{H}\left(H_{B}\right) \in R^{2 \times(k+m)}$ is the matrix formed on the basis $H_{B}$.

In this case, multiplicative adaptive algorithm gives only the minimization of the prediction error. This approach can be applied when high accuracy of parametric estimation is not needed.

\section{Case of interdependence between lags}

Consider a case of an existence of interdependence between lag variables. In this case a method of principal components [30] to identification of parameters of system applies. The method of principal components is a transformation of input variables for interdependence elimination between them. It demands the implementation of a some of the preliminary stages and is not always applicable in actual practice.

Remark 1. A method of adaptive expectations [19] for construction of regressions and autoregressive models often apply. It on obtaining of the forecast an input variable on the basis of the exponential smoothing filter (the recursive filter of first order) is grounded. The exponential smoothing filter is not adaptive filter.

We apply an approach based on transformation the of input lag variables to exclude the interdependence between them. New variables are a difference between lag variables $x_{i, j, n}, x_{i, j+1, n}$

$$
z_{i, n}=x_{i, j, n}-x_{i, j+1, n},
$$

where $x_{i, j, n}$ is a component of a vector $X_{i, n}$.

Remark 2. Some authors [4] this approach to exclude the correlation between the lags of the dependent variable are applied. Such approach is valid at an estimation of parameters on the basis of SLM. The model obtained in it a case, is not dynamic. It should be considered at a choice of adequate methods of identification.

Choice a component of a vector $X_{i, n}$, subject to transformation, depends on properties of a system depends. Such a transformation to variables with a large value of lag applies.

The technique of adaptive identification in this case does not differ from the approach stated in section 3.A.

\section{ADAPTIVE IDENTIFICATION OF SYSTEM WITH LAG ON $X_{y, n}$}

Consider the case of the presence of lagged variables in the output variable of the system (1). We the relationship between the elements of the vector $X_{y, n}$ are not considered. We in section 3 have considered existing approaches. We will consider a case of presence correlation between an output of the system (1) and disturbance $\hat{\xi}_{n}$.

Let $X_{n}=X_{y, n}, X_{y, n}=\left[y_{n-1}, \ldots, y_{n-k}\right]^{T}$. This means that the system is described by the autoregressive equation (1). Let disturbance $\xi_{n} \in R$ is linked with an output $y_{n}$ a linear dependence. Apply to identification of parameters of system (1) model

$$
\hat{y}_{\xi, n}=\hat{A}_{n-1}^{T} U_{n}+\hat{B}_{n-1}^{T} X_{\hat{y}, n}+\hat{\xi}_{n},
$$

where $\hat{\xi}_{n} \in R$ is an estimation of disturbance, $\hat{A}_{n}, \hat{B}_{n}$ are vectors of adjusted parameters, $X_{\hat{y}, n}=\left[\hat{y}_{\xi, n-1}, \ldots, \hat{y}_{\xi, n-k}\right]^{T}$ is a vector formed on the basis of an output model $\hat{y}_{\xi, n}$.

For system (1) we have informational set $\mathrm{I}_{o}$.

Problem: on the basis of the analysis $\mathrm{I}_{o}$ by means of model (26) estimate set of parameters system (1) and obtain estimations of disturbance $\xi_{n}$, minimizing criterion $V_{e, n}$.

We show how to determine interdependence between $y_{n}$ and $\xi_{n}$. We modify the procedure, offered in [26].

Algorithm $A R_{\xi}$.

1. Generate a variable $s_{n}=I^{T} U_{n}$, apply model

$$
\hat{y}_{s, n}=\hat{a}_{s, 0}+\hat{a}_{s, 1} s_{n} .
$$

Define parameters of model by means of SLM.

2. Introduce a variable $\varepsilon_{n}=\hat{y}_{s, n}-y_{n}$. Apply model

$$
\gamma_{\varepsilon, x_{i, n}}=a_{\varepsilon, i, 0}+a_{\varepsilon, i, 1} x_{i, n}, 1 \leq i \leq m
$$

and define its parameters with help SLM and coefficients of determinations $r_{\varepsilon, x_{i, n}}^{2}$.

3. Set level $\delta_{x}>0$ and from $x_{i, n} \in X_{n}$ for which the condition is satisfied $r_{\varepsilon, x_{i, n}}^{2} \geq \delta_{x}$, generate a vector $\tilde{X}_{n}$ (it should coincide with $X_{n}$ ).

4. Construct model $\hat{y}_{\varepsilon, n}=A_{\varepsilon}^{T} \tilde{X}_{n}$ for $\varepsilon_{n}$ and define an error $\varepsilon_{\varepsilon, n}=\varepsilon_{n}-\hat{y}_{\varepsilon, n}$. Compute $r_{\varepsilon_{\varepsilon}, y_{n}}^{2}$. If $r_{\varepsilon_{\varepsilon}, y_{n}}^{2} \geq \delta_{\varepsilon, y}$, where $\delta_{\varepsilon, y}>0$ is a specified value, then is a relationship between $y_{n}$ and $\xi_{n}$. 
In the conditions of a priori uncertainty construct the observer $\xi_{n}$ for the account of existing interdependence. Designate an error of prediction $y_{n}$ by means of model (26) as $e_{\xi, n}=y_{n}-\hat{y}_{\xi, n}$. Let $\hat{\xi}_{n}=\hat{\chi}_{n-1} e_{\xi, n-1}$. Write (26) in a form

$$
\hat{y}_{\xi, n}=\hat{A}_{n-1}^{T} U_{n}+\hat{B}_{n-1}^{T} X_{\hat{y}, n}+\hat{\chi}_{n-1} e_{\xi, n-1},
$$

where $\hat{\chi}_{n-1}$ is the adjusted parameter.

This approach is applicable when the error $e_{\xi, n}$ is the range of variation preserves. The range of a change $e_{\xi, n}$ in the adaptation process is not constant. Therefore contain the distorted information about a disturbance.

We will use variables $\varepsilon_{n}$ or $\varepsilon_{\varepsilon, n}$ as sources about disturbance. We will rewrite (27) as

$$
\hat{y}_{\xi, n}=\hat{A}_{n-1}^{T} U_{n}+\hat{B}_{n-1}^{T} X_{\hat{y}, n}+\hat{\chi}_{n-1} \varepsilon_{n} .
$$

The equation for a prediction error $y_{n}$

$$
e_{\xi, n}=\delta A_{n-1}^{T} U_{n}+\delta B_{n-1}^{T} X_{\hat{y}, n}+\delta \xi_{n},
$$

where $\delta \xi_{n}=\xi_{n}-\hat{\chi}_{n-1} \varepsilon_{n}$.

Apply Lyapunov second method with the function $V_{e_{\xi}, n}=e_{\xi, n}^{2}$ and obtain adaptive algorithms

$$
\begin{aligned}
& \hat{A}_{n}=\hat{A}_{n-1}+\Gamma_{A} e_{\xi, n} U_{n}, \\
& \hat{B}_{n}=\hat{B}_{n-1}+\Gamma_{B} e_{\xi, n} X_{y, n}, \\
& \hat{\chi}_{n}=\hat{\chi}_{n-1}+\gamma_{\chi} e_{\xi, n} \varepsilon_{n},
\end{aligned}
$$

where $\Gamma_{A}, \Gamma_{B}, \gamma_{\chi}$ are the matrixes ensuring convergence of algorithms.

From (30) - (32) we obtain the equations for $\delta A_{n}$, $\delta B_{n}$. They are described by the equation (9) with $e_{n}=e_{\xi, n}$. For $\delta \hat{\chi}_{n}=\chi-\hat{\chi}_{n}$ we have the equation

$$
\delta \chi_{n}=\delta \chi_{n-1}-\gamma_{\chi} e_{\xi, n} \varepsilon_{n}
$$

where $\chi$ is a number ensuring equality $\xi_{n}=\chi \varepsilon_{n}$.

The equation (33) is true not for all $\varepsilon_{n}$. But now we consider that this relation is fulfilled.

Theorem 3. Let conditions of the theorem 1 are satisfied, and also $\left|\varepsilon_{n}\right| \leq \mu<\infty$ for $\forall n \geq 0$. Then algorithms (30) (33) converge, if

$$
2 \pi \geq \bar{\gamma}^{2} \beta^{2}+\gamma_{\chi}^{2} \mu^{2}
$$

where $\pi=\min \left(\tilde{\gamma}, \gamma_{\chi}\right)$.

Proof. Consider LF $V_{\delta, n}=\left\|\delta H_{n}\right\|^{2}+\left(\delta \chi_{n}\right)^{2}$. We obtain for $\Delta V_{\delta, n}$

$$
\begin{aligned}
\Delta V_{\delta, n}= & -2 e_{\xi, n}\left(\delta H_{n-1}^{T} \Gamma K_{n}+\gamma_{\chi} \delta \chi_{n-1} \varepsilon_{n}\right) \\
& +e_{\xi, n}^{2} K_{n}^{T} \Gamma^{2} K_{n}+e_{\xi, n}^{2} \gamma_{\chi}^{2} \varepsilon_{n}^{2} .
\end{aligned}
$$

Apply conditions of the theorem 3 and $\Delta V_{\delta, n}$ transform to the form

$$
\Delta V_{\delta, n} \leq-2 \pi e_{\xi, n}^{2}+e_{\xi, n}^{2} \bar{\gamma}^{2} \beta^{2}+e_{\xi, n}^{2} \gamma_{\chi}^{2} \mu^{2}
$$

Algorithms (9), (33) converge, if

$$
2 \pi \geq \bar{\gamma}^{2} \beta^{2}+\gamma_{\chi}^{2} \mu^{2}
$$

Consider now a case when $\xi_{n}=\chi \varepsilon_{n}$ is not fulfilled. This situation is standard in many cases. Therefore we suppose that

$$
\delta \xi_{n}=\xi_{n}-\hat{\chi}_{n-1} \varepsilon_{n}=\delta \chi_{n-1} \varepsilon_{n}+\varepsilon_{\chi}
$$

where $\left|\varepsilon_{\chi}\right| \leq \mu_{\chi}, \mu_{\chi} \geq 0$ is some number.

$\varepsilon_{\chi}$ is the uncertainty arising from the absence of information about the perturbation. In this case properties of system identification by means of the theorem 2 can be estimated.

Parameter $\gamma_{\chi}$ in (33) we find from the condition

$$
\min _{\gamma_{\chi}} e_{\xi, n}^{2} \rightarrow \gamma_{\chi}^{*}
$$

So, methods of adaptive identification of discrete systems with lag variables are considered. Effect of disturbance on properties of system identification is researched.

\section{RESUlts OF SimUlation}

\section{A. Modelling case}

Consider system (1) with $A=[2.5,2.4,2]^{T}$ and $B=[0.8,0.4]^{T} ; U_{n} \in R^{3}, u_{i, n}(i=1,2)$ is a random variable having a final expectation and a variance; $X_{n}=\left[u_{1, n-1}, u_{1, n-2}\right]^{T} ; \xi_{n}$ is the random function having zero expectation and a final variance, $\left|\xi_{n}\right| \leq 0.3$.

The input $u_{1, n}$ is obtained as a solution of a difference equation with the random input having a nonzero expectation and a final variance.

The results of the adaptive identification system with 
$\xi_{n}=0$ are presented in Fig. 1. We do not see because of the presence of the interdependence between the elements of the vector $X_{n}$ of convergence the adaptive algorithms (7), (8). But trajectories of system of identification are restricted.

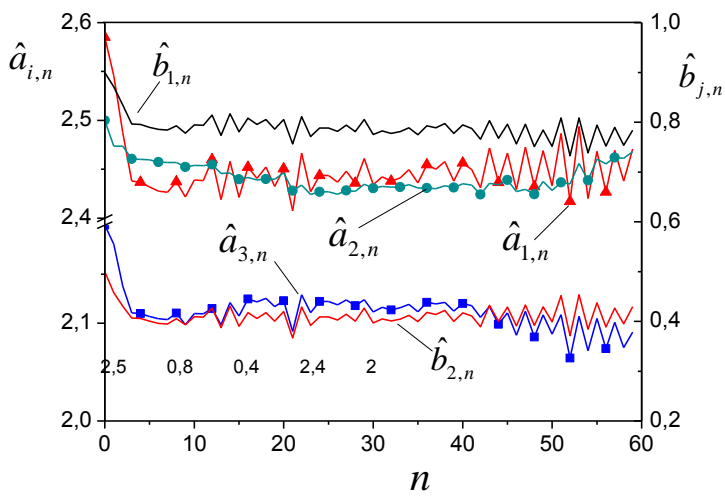

Fig.1. Tuning of parameters system.

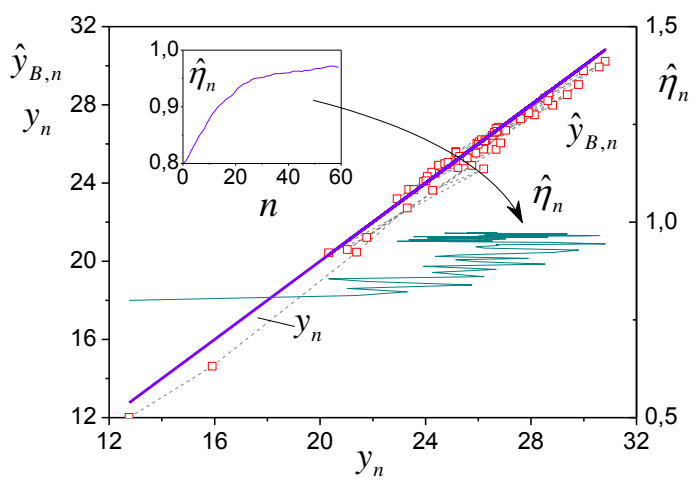

Fig.2. Results of model identification (21).

On Fig. 2 we showed results of adjustment of multiplicative parameter $\hat{\eta}_{n-1}$ and adequacy of model (21). The approach described in section 3.B is applied to model identification. We have defined a vector $H_{B}=\left[A_{B}, B_{B}\right]^{T}$, applied algorithm $A H_{B}$, where $A_{B}=[2.48,2.05,1.8]^{T}$, $B_{B}=[0.83,0.7]^{T}$. In the condition (20) we used following magnitudes $\Xi_{y_{B}}=691, \quad \Xi_{y}=660, \quad r_{y, y_{B}}^{2}=0.98$, $\bar{y}=25, \bar{y}_{B}=26.1, \varphi=1.5, \varphi_{B}=35$. Next, apply the algorithm (22) with $\gamma_{\eta}=0.0008$. Explain the character of a change $\hat{\eta}_{n-1}$ compensating of parametric disturbances because of vector choice $H_{B}$. The maximum error of prediction $e_{\eta, n}$ on the interval $[24,32]$ a change $y_{n}$ does not exceed $2.5 \%$.

The approach described to section 3.C was applied to the improvement of the quality of parametric identification. We instead of $x_{2, n}$ the variable $z_{1, n}=x_{1, n}-x_{2, n}$ used Results of work of adaptive algorithms (7), (8) shown on Fig. 3. Matrixes $\Gamma_{A}, \Gamma_{B}$ in (7), (8) are equal
$\Gamma_{A}=\operatorname{diag}(0.005 ; 0.008 ; 0.008), \Gamma_{B}=\operatorname{diag}(0.02 ; 0.008)$.

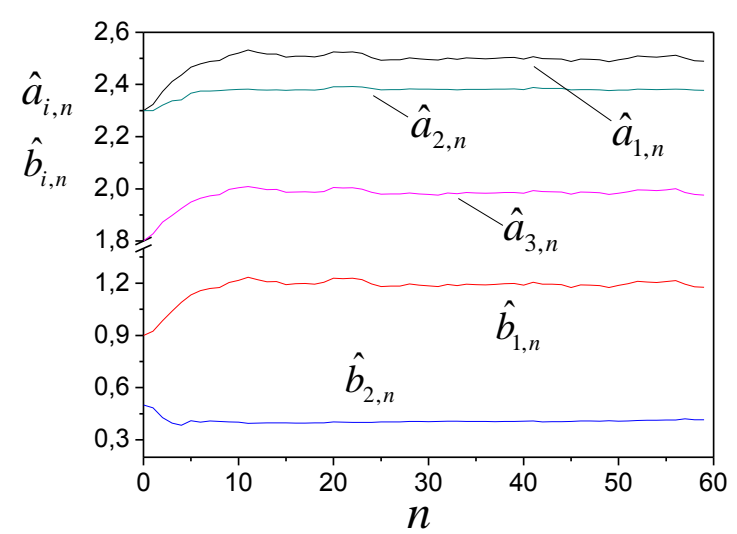

Fig.3. Tuning of parameters of model (3).

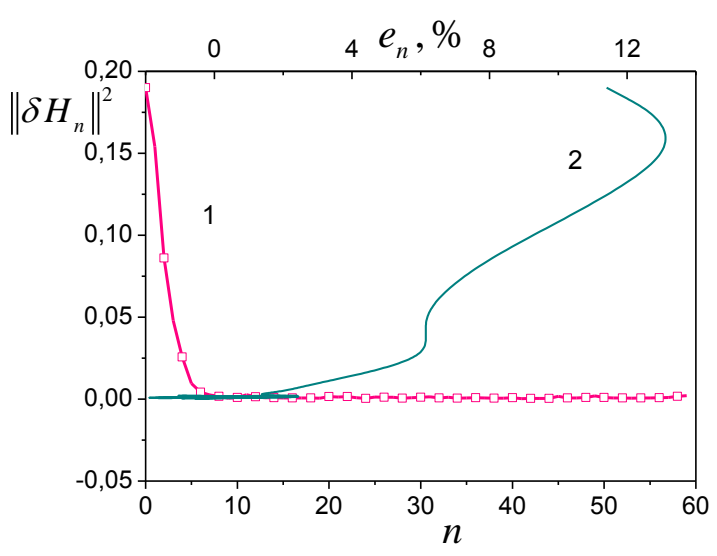

Fig.4. Change $\left\|\delta H_{n}\right\|^{2}$.

Changing norms parametric errors

$$
\left\|\delta H_{n}\right\|^{2}=\left\|\delta A_{n}\right\|^{2}+\left\|\delta B_{n}\right\|^{2}
$$

show in Fig. 4.

On Fig. 4 designation 1 is a change $\left\|\delta H_{n}\right\|^{2}$ as the function from $n$, and the designation 2 is a change $\left\|\delta H_{n}\right\|^{2}$ as the function from $e_{n}$.

The offered approach gives true estimations of examined system. The maximum prediction error for $n \geq 10$ is less than $2 \%$. The correct choice of the components of the vector $X_{i, n}$ in (3) confirm the values of the coefficients of determination between $z_{1, n}$ and $u_{1, n}, x_{1, n}: r_{z_{1}, u_{1}}^{2}=0$, $r_{z_{1}, x_{1}}^{2}=0.028$.

Consider now system (1) with a lag on $y_{n}$ : $X_{y, n}=y_{n-1}$. Let

$$
B=b=0.4, A=[2 ; 2.6 ; 1.8]^{T}, \xi_{n}=\xi\left(y_{n}\right) .
$$

Apply model (28) to system identification with the I.J. Intelligent Systems and Applications, 2016, 3, 1-13 
modified adaptive algorithms (30) - (32)

$$
\begin{gathered}
\hat{A}_{n}=\hat{A}_{n-1}+\frac{\Gamma_{A} e_{\xi, n} U_{n}}{\left\|U_{n}\right\|^{2}+y_{n-1}^{2}+\varepsilon_{n}^{2}}, \\
\hat{b}_{n}=\hat{b}_{n-1}+\frac{\Gamma_{B} e_{\xi, n} y_{n-1}}{\left\|U_{n}\right\|^{2}+y_{n-1}^{2}+\varepsilon_{n}^{2}}, \\
\hat{\chi}_{n}=\hat{\chi}_{n-1}+\frac{\gamma_{\chi} e_{\xi, n} \varepsilon_{n}}{\left\|U_{n}\right\|^{2}+y_{n-1}^{2}+\varepsilon_{n}^{2}} .
\end{gathered}
$$

Modification does not change property of procedures (30) - (32). But it simplifies choice of matrixes $\gamma_{\chi}, \Gamma_{A}$, $\Gamma_{B}$.

Matrixes are equal

$$
\Gamma_{A}=0.36 I_{3}, \Gamma_{B}=0.06, \gamma_{\chi}=0.15
$$

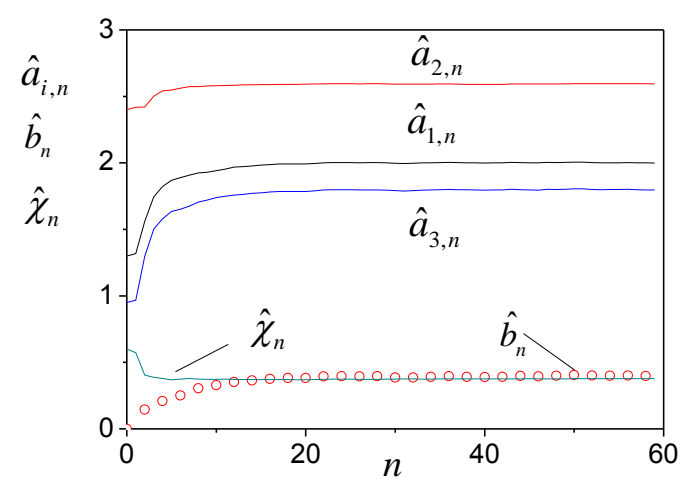

Fig.5. Tuning of parameters of model (28).

Results of work of algorithms (35) - (37) show in Fig. 5. On Fig. 6 we have presented an estimation of disturbance $\xi_{n}$ and a change $\left\|\delta A_{n}\right\|^{2}$. The error of prediction $e_{\xi, n}$ with $n \geq 30$ does not exceed $6 \%$.

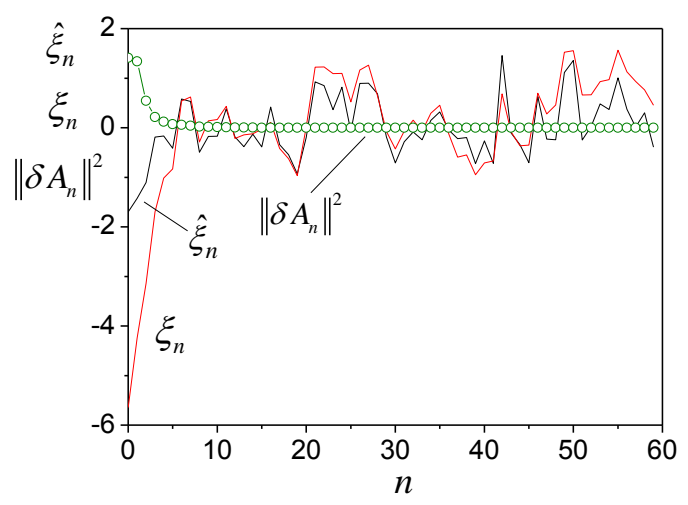

Fig.6. Estimation of disturbance and norm of parametric misalignment.

Consider the same system no registering interdependence between $y_{n}$ and $\xi_{n}$. Substitute $X_{\hat{y}, n}=\hat{y}_{n-1}$ in (29) on $X_{y, n}=y_{n-1}$. We instead of an autoregressive model have regression model. Such approach is dominating in the analysis of lag models. Application of the offered adaptive algorithms in this case is impossible.

\section{B. Synthesis of models economy}

Consider the data on the cores macroeconomic and tax parameters of the Russian Federation for 2004-2012. We analyze following parameters: $u_{1}$ is gross domestic product (milliard. roubles) (GDP), $u_{2}$ is export (milliard. roubles), $u_{3}$ is import (million roubles), $u_{4}$ is employment of population (million people), $u_{5}$ is a consumer price index $(\%), u_{6}$ is receipts of the tax to the added cost (VAT) (million roubles).

Output parameters are GDP $\left(y_{1}=u_{1}\right)$ and import $\left(y_{2}=u_{3}\right)$. The choice of model structure describing the change of these indicators, described in [26, 31].

1. Model for prediction GDP. To description of change GDP apply adaptive model

$$
\hat{y}_{1, n}=\hat{b}_{1, n-1} \hat{y}_{1, n-1}+\hat{a}_{2, n-1} u_{2, n}+\hat{a}_{3, n-1} u_{3, n}+\hat{b}_{2, n-1} u_{2, n-1} .
$$

We applied the algorithms (7), (8) to adjust the parameters of the model (38). Matrixes $\Gamma_{A}$ and $\Gamma_{B}$ are equal

$$
\begin{gathered}
\Gamma_{B}=\operatorname{diag}(0,00007 ; 0,00001), \\
\Gamma_{A}=\operatorname{diag}(0,000021 ; 0,000018) .
\end{gathered}
$$

The results of the tuning of the model parameters are show on Fig. 7. The model has the form

$$
\hat{y}_{1, n}=0,76 \hat{y}_{1, n-1}+0,02 u_{2, n}+0,074 u_{3, n}-0,03 u_{2, n-1} .
$$

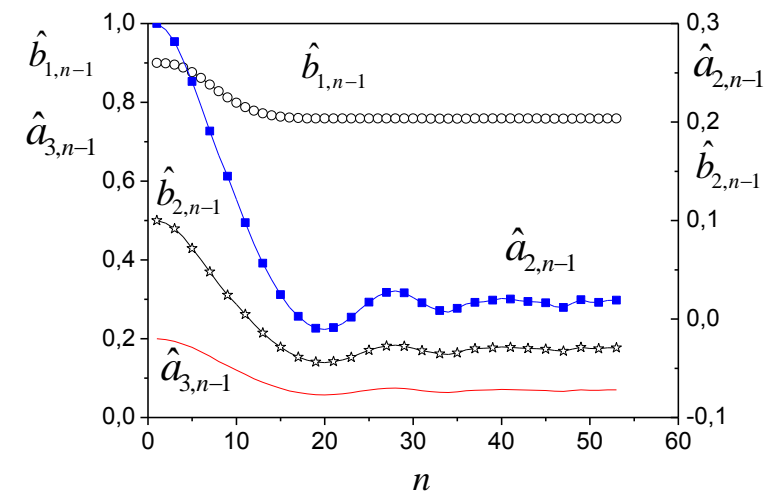

Fig.7. Tuning of parameters model (38).

The output of the model (38) and GDP is presented in Fig. 8. Maximum value of a relative error of prediction for $n \geq 30$ does not exceed $4 \%$. The coefficient of determination is 0.99 . 


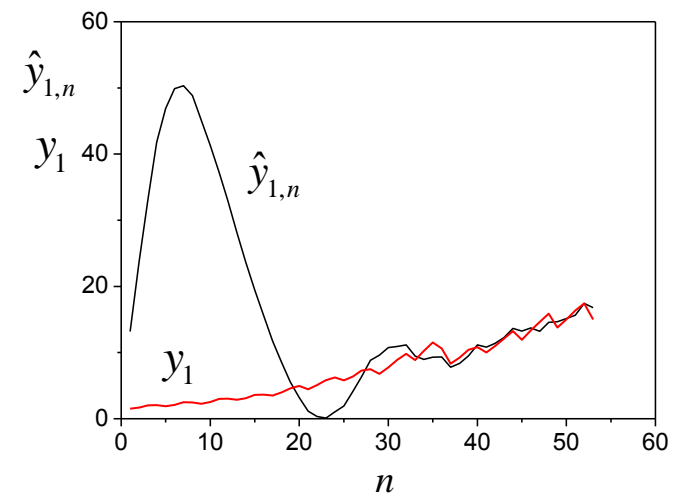

Fig.8. Estimation of adequacy model.

2. Model for import prediction. Determine the relationship between the volume of imports and exports products to estimate state of economy. Such relationship exists and is described by the nonlinear adaptive model

$$
u_{3, n}=\hat{a}_{n-1} u_{2, n}^{\hat{\beta}_{n-1}}
$$

where $\hat{a}_{n-1}, \hat{\beta}_{n-1}$ are adjusted parameters of model.

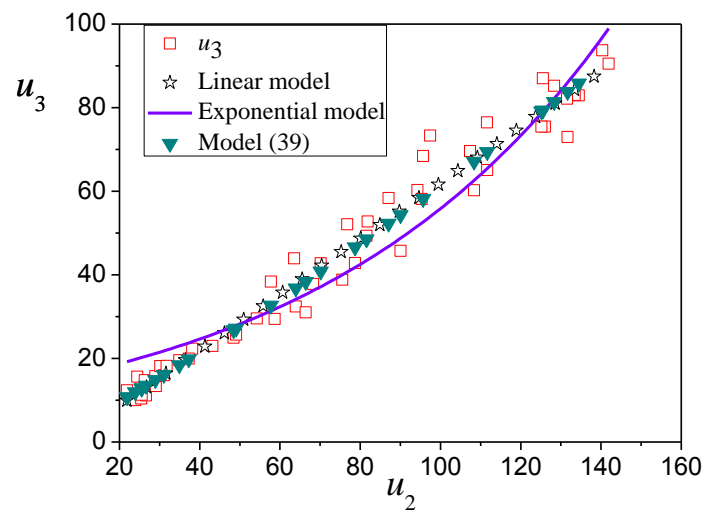

Fig.9. Analysis of the quality model (39)

We do not discuss adaptive algorithms for estimation of model parameters. They are modification of procedures (7), (8). Estimations for model parameters are equal: $\hat{a}_{n-1}=0,319, \hat{\beta}_{n-1}=1,14$. The coefficient of determination is 0,975 . The model study (39) displays that import is almost a third of export inflows of the country.

Show the results of model (39) in Fig. 9. We on Fig. 9 compare the effectiveness of model (39) to linear and exponential models. These models have the form

$$
u_{3, n}=9.97 e^{0.017 u_{2, n}}, u_{3, n}=0.67 u_{2, n}-4.65
$$

Coefficients of determinations for linear and exponential models are 0.96 and 0.92 . On linear model import is two thirds of export. This result correcting on magnitude 4.65. We obtain the statement made above about relationship $u_{2}$ and $u_{3}$.
We are correcting this result, considering a model absolute term.

3. Prediction of volume of arrears on loans. We analyzed the data of the Central Bank Russia for 2014.

We used the following factors [32] influencing volume of arrears on loans legal bodies and individual businessmen $p_{n}$ :

i) the delayed backlog under credits of legal bodies and individual businessmen, million roubles $\left(u_{3}\right)$;

ii) residual of attracted means of clients not credit organizations, million roubles $\left(u_{6}\right)$;

iii) backlog under credits legal bodies and individual businessmen, million roubles $\left(u_{8}\right)$.

We have obtained regression model $P S$

$$
\hat{p}_{s, n}=325578,33+13453,06 u_{3}-0,02 u_{6}+0,05 u_{8} .
$$

The coefficient of determination is equal $r_{s}^{2}=0,97$. Practical application of the obtained model is limited. Static models to predict economic indicators are rarely used. Explain it the necessity of development of prediction procedure. The essential shortage of model (40) is large value of a standard error (SE). SE for model (40) is $s e_{s}=20731,93$. This explain the fluctuations in the output of the model $\hat{p}_{n}$ relative to the index $p_{n}$.

We synthesized model $P L$ with a lag on $p_{n}$ for decrease SE

$$
P L: \begin{aligned}
\hat{p}_{l, n}= & 0.67 \hat{p}_{l, n-1}+120278.02 \\
& +8693.25 u_{3}-0.01 u_{6}+0.02 u_{8} .
\end{aligned}
$$

The coefficient of determination is $r_{l}^{2}=0.99$, and the standard error almost on $70 \%$ has decreased: $s e_{l}=14155.78$. Show work of the obtained models on Fig. 10.

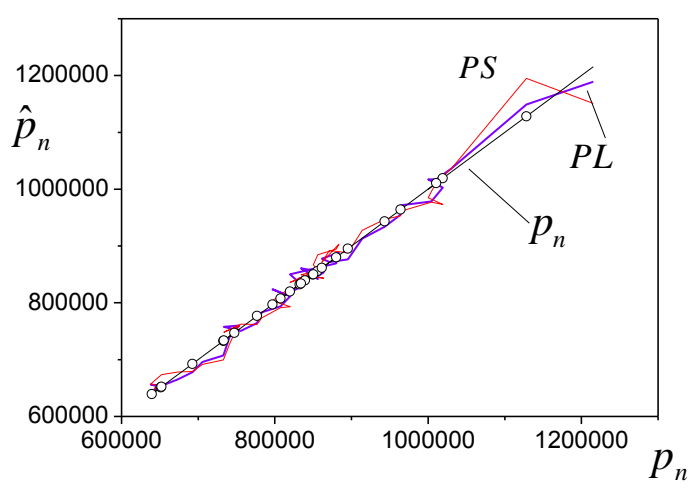

Fig.10. Forecasting properties of models (40), (41).

We see that the model (40) is not always adequately 
current value $p_{n}$ describes. This is true at large values $p_{n}$. Accounting lag (model (41)) improves the accuracy of prediction.

\section{CONCLUSION}

Adaptive algorithms of parametric identification discrete systems with lag variables are offered. The case of the presence lag input variables is considered. Adaptive algorithms (AA) are designed. Convergence AA and boundedness of trajectories adaptive system for this class of systems are proved. Convergence domain AA depends on operating disturbances. In systems where low demands are made to the quality of work of system a parametric estimation, multiplicative adaptive algorithms are offered. Procedure of choice of a vector of base parameters of multiplicative model is developed. The working capacity of the adaptive system with a multiplicative model is proved. The choice of parameters the multiplicative identification of conditions to minimize the prediction error criterion executes.

The analysis of work adaptive algorithms identification systems with a distributed lag on an output variable is given in the second part of paper. The case presence of linear interdependence between an output of a system and operating disturbance is studied. The problem solution is based on an estimation of operating disturbance. Corresponding procedures are designed and their working capacity is proved. We do not consider identification procedure when there is a correlation between lags of an output system. In this case, we apply the methods developed in the second part of the work.

Results of simulation which confirm working capacity of adaptive methods are presented. Models of prediction tax parameters of the Russian Federation are developed.

\section{REFERENCES}

[1] N. Gershenfeld, The nature of mathematical modelling. Cambridge: Cambridge University Press, 1999.

[2] T. Kailath, "Linear Least Squares Estimation," Benchmark Papers in Electrical Engineering and Computer science. Dowden, Hutchinson \&Ross, 1977.

[3] N.N. Karabutov, Structural Identification of Systems. Analysis of Information Structures. Moscow: URSS/ Librokom, 2009.

[4] E. Malinvaud, Statistical methods in econometrics. 3d ed. Amsterdam: North-Holland Publishing Co. 1980.

[5] J. Johnston, Econometric methods. 2nd edition. New York: McGraw-Hill Book Company, 1972.

[6] I.C. Demetriou, E.E. Vassiliou, "An algorithm for distributed lag estimation subject to piecewise monotonic coefficients," International Journal of Applied Mathematics, 2009, vol. 39, no. 1, pp. 1-10.

[7] P.J. Dhrymes, Distributed Lags: Problems of Estimation and Formulation. San Francisco: Holden-Day, 1971.

[8] B. Armstrong, "Models for the relationship between ambient temperature and daily mortality," Epidemiology, 2006, 17(6), pp. 624-631.

[9] C.R. Nelson, G.W. Schwert, "Estimating the parameters of a distributed lag model from cross-section data: the case of hospital admissions and discharges," Journal of the American Statistical Association, 1974, vol. 69, no. 347, pp. 627-633.

[10] A. Gasparrini, B. Armstrong, M.G. Kenwardb, "Distributed lag non-linear models," Statistics in Medicine. 2010, 29(21), pp. 2224-2234.

[11] L.M. Коуск, Distributed Lags and Investment Analysis. Amsterdsm: North-Holland Publishing Company, 1954.

[12] Fisher, "Note on a short-cut method for calculating distributed lags," Bulletin de l'Institut International de Statistique, 1937, vol. 29.

[13] P.J. Dhrymes, L.R. Klein, K. Steiglitz. "Estimation of Distributed Lags," International Economic Review, 1970, vol. 11, no. 2, pp. 235-250.

[14] S. Almon, "The distributed lag between capital appropriations and expenditures," Econometrica, 1965, vol. 33, pp. 178-196.

[15] H. Theil, R.M. Stern, "A simple unimodal lag distribution," Metroeconomica, 1960, vol. 12, pp. 111-119.

[16] R. Solow, "On a family of lag distributions," Econometrica, 1960, vol. 28, pp. 393-406.

[17] D.W. Jorgenson, "Minimum variance, linear, unbiased seasonal adjustment of economic time series," Journal of the American Statistical Association, 1964, vol. 59, no. 307, p.p. 681-724.

[18] W.H. Greene, Econometric analysis, 7th ed. New Jersey: Prentice Hall Pearson Education, Inc., 2012.

[19] G.C. Chow, "Usefulness of adaptive and rational expectations in economics," CEPS Working Paper No. 221, Princeton University, 2011.

[20] J.H. McCulloch, "Adaptive least squares estimation of the time-varying Taylor rule," 2007. www.econ.ohiostate.edu/jhm/papers/TaylorALS.pdf.

[21] T.F. Cooley, E.C. Prescott, "Tests of an Adaptive Regression Model," The review of economics and statistics, The MIT Press, 1973, vol. 55, no. 2, pp. 248-256.

[22] R. Butler, J. McDonald, R. Nelson, and S. White, "Robust and Partially Adaptive Estimation of Regression Models," Review of Economics and Statistics, 1990, 72, pp. 321327.

[23] C. Bouveyron, P. Gaubert, J. Jacques, “Adaptive regression models for modeling and understanding evolving populations," CS-BIGS, 2011, vol. 4(2), pp. 83-92.

[24] I.C. Demetriou, E.E. Vassiliou, "An algorithm for distributed lag estimation subject to piecewise monotonic coefficients," International journal of applied mathematics, 2009, vol. 39, no. 1, pp. 1-10.

[25] N.N. Karabutov, "Structural identification of static systems with distributed lags," International journal of control science and engineering, 2012, vol. 2, no. 6, pp.136142.

[26] N.N. Karabutov, "Structural identification of systems with distributed lag," International journal of intelligent systems and applications, 2013, vol. 3, no. 11, pp. 1-10.

[27] N.N. Karabutov, "Adaptive Prediction Model of Tax Indicators," Economy. Taxes. Right, 2014, no. 6, pp. 101-110.

[28] N.N. Karabutov, Adaptive identification of system: information synthesis. Moscow: URSS, Komkniga, 2006.

[29] E.A. Barbashin, Lyapunov Function. Moscow: Nauka, 1970.

[30] N.R. Draper, H. Smith, Applied regression analysis. Second edition. New York: John Wiley \& Sons, Inc., 1981.

[31] N.I. Karabutov, V.G. Feklin, "Mathematical models of tax index forecasting," Economy. Taxes. Right, 2016, no. 6, pp. 115-121.

[32] N.I. Karabutov, V.G. Feklin, "Forecasting models the volume of overdue debt on loans," Bulletin Financial University, 20015, no.4, pp. 116-121. 


\section{Authors' Profiles}

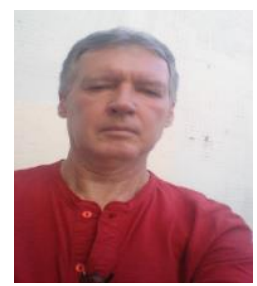

Nikolay Karabutov is the professor of department Problems Control of Moscow state engineering university of information technology, radio engineering, and electronics. Doctor of technical sciences, professor.

Nikolay Karabutov is the professor of department Problems Control of Moscow state engineering university of information technology, radio engineering, and electronics. Doctor of technical sciences.

How to cite this paper: Nikolay Karabutov, "System with Distributed Lag: Adaptive Identification and Prediction", International Journal of Intelligent Systems and Applications (IJISA), Vol.8, No.3, pp.1-13, 2016. DOI: 10.5815/ijisa.2016.03.01 